\title{
SOMATIC DISEASE AND PSYCHOLOGICAL DISORDER
}

\author{
PETER F. M. VERHAAK \\ (Received 5 September 1996; accepted 6 September 1996)
}

\begin{abstract}
The association between physical and psychological disorders has been demonstrated repeatedly. There are a number of explanations for this association, each of them pointing to specific diseases and operationalizations of mental distress. In this article, the relationship between various somatic diseases and a number of indices for psychological distress was investigated. Within one study population. patients with different somatic diseases were identified, and their experience with mental distress, their requests for help from their GP during consultations, and their GPs diagnoses were registered and compared with the total study population: It appears that relationships could be demonstrated between experience of distress and presentation of psychological symptoms during consultations, on the one hand. and common physical disorders, on the other. Patients with neurological diseases (Parkinson's. epilepsy, multiple sclerosis) and gastric ulcers showed the same relationships, but were also more frequently diagnosed by the GP as having psychological disorders. Patients with a number of other serious somatic diseases. such as diabetes, cancer, and arthritis, did not distinguish themselves in a positive way on one of indices for psychological distress. @1 1997 Elsevier Science Inc.
\end{abstract}

Keywords: Chronic disease; Epidemiology: General practice: Psychiatry: Psychological symptoms.

\section{INTRODUCTION}

Somatic diseases and psychological disorders are closely interrelated. On the one hand, patients suffering from mental illness report more physical complaints than "average" patients when visiting a doctor [1-4], and many psychiatric out-patients and hospitalized psychiatric patients are also physically ill [5, 6]. Even mortality risks are considerably higher in patients with psychological distress than in other patients $[7,8]$.

On the other hand, however, high prevalences of psychological disorders. especially depression and anxiety, have been reported among patients who suffer from somatic chronic disease [9-14], although not all studies are in agreement with this. For instance, Cassileth et al. [15], who studied 758 patients with arthritis, diabetes, cancer, renal disease, and dermatological disorders, found that their patients did not differ from average patients with respect to anxiety, depression, general positive affect, emotional ties, and loss of control. They concluded that psychological adaptation among patients with chronic illness is remarkably effective and is fundamen-

NIVEL, Netherlands Institute of Primary Health Care. Utrecht, The Netherlands.

Address correspondence to: Peter F. M. Verhaak. Netherlands Institute on Primary Health Care. P.O. Box 1568, 3500 BN Utrecht. The Netherlands. Tel: 313023199 46: Fax: 313023192 90; E-Mail: p.verhaak@nivel.nl 
tally independent of a specific diagnosis. Spitzer et al. [16] reported no significant deviations in mental health or social functioning among patients with a number of somatic diseases either.

There are a number of possible explanations for the relationship between somatic disease and psychological disorder. Each of them is associated with specific manifestations of disease and psychological disorder.

In some instances, there is evidence for a direct biological link between a somatic disease and a psychological disorder, especially depression. Some diseases may cause anatomical or physiological changes, which give rise to feelings of depression or anxiety. For example, Brown and Paraskevas [17] suggest that depression in pancreatic patients could be caused by immunological interference with the activity of serotonin. Cerebral vascular accidents can cause depression, dependent on the location of the lesions. Parkinson's disease and multiple sclerosis are also central nervous system disorders which are thought to have a direct biological link with depression (see Miranda et al. [18] for an overview). It should be stressed that these direct biological links have been demonstrated for a limited number of well-defined somatic diseases and explain a small minority of prevalent psychological disorders.

In the absence of a direct biological link, there are a number of other explanations for the relationship between psychological disorder and somatic disease. Some of these explanations consider psychological disorders to be a result of physical disease, whereas others consider physical illness to be a result of mental distress. In the former, chronic illness is considered a stressor, causing psychological distress and resulting in help-seeking behavior. Following this explanation, one should expect somatic diseases to cause greater mental distress as they become more severe, more life-threatening, and more limiting to the functioning of the patient.

There are several explanations for physical illness being a result of psychological disorder. First, psychological disorder may be expressed by physical symptoms, leading to somatic diagnoses. For example, the DSM diagnoses of affective disorders include a number of neurovegetative symptoms that might easily be diagnosed as somatic disorders [19]. Second, psychological stress, or a depressed or anxious personality [20], have been implicated as the cause of gastric ulcers, coronary heart disease, migraine, and musculoskeletal disorders such as low back pain. Third, and especially with severe psychiatric disorders, somatic illness might be caused by neglect, poor health behaviors, and noncompliance to somatic regimens. For example, Hayward [21] pointed to strong evidence that mental illness is associated with elevated rates of cigarette smoking, physical inactivity, hypertension and hypercholesterolaemia. The somatic diseases that are considered the result of mental distress are partly ill-defined conditions and partly the formerly so-called "psychosomatic disorders," such as migraine, gastrointestinal disorders, etc.

The last group of explanations for the relationship between psychological disorder and somatic disease concern the phenomenon of comorbidity. With increasing age, the risk of somatic illness [22] and psychological disorder [23] both increase. This explanation appears to be especially valid for age-related diseases, such as rheumatoid arthritis or diabetes.

Most of the aforementioned research has focused on somatic diseases in general or on one specific disease, such as cancer, diabetes, or coronary heart disease. Thus, diseases that may have a different impact on psychological disorders (or vice versa) 
have been grouped together. Comparisons between various somatic diseases are rare. In addition, almost all of the research has limited itself to a narrow concept of psychological disorder, namely psychiatric disorder as measured by screening or diagnostic instruments. Thus, the psychological disorder is defined by professionals, irrespective of the patient's evaluation of his or her mental distress. Help-seeking behavior and demands for help by the patient are underevaluated aspects of the mental distress experienced by physically ill patients.

By relating different diseases, all taken together, to psychiatric disorder the resulting relationship might obscure a number of more differentiated relationships. Therefore, in this article, a number of specified somatic diseases will be considered separately and related to a number of psychological parameters, including diagnoses as well as patients' demands for help. More specifically, the following aspects of psychological disorder will be considered:

- The experience of mental distress (patient).

- Psychological demands for help (patient).

- Demands for help concerning fear, anxiety, and concern about a disease (patient).

- Diagnoses by the general practitioner (GP).

- Attributions by the general practitioner (GP).

Patients suffering from each disease will be compared in these five aspects with patients not suffering from that disease.

The following questions will be answered: Are there differences between patients with different somatic diseases with regard to their mental distress, their demands for help for their psychological problems, and the psychological diagnoses and attributions made by GPs?

\section{METHODS}

Data were derived from the Dutch National Study of Morbidity and Interventions in General Practice. During this nationwide survey, 161 GPs recorded all their doctor-patient contacts over a period of 3 months. Participating practices were randomly selected according to a stratification procedure that guaranteed a sufficient number of practices from each region and each level of urbanization.

\section{Dataset 1}

A random sample of patients older than 14 years $(N=10,787)$ was interviewed. One hundred patients registered at the practice of each participating GP were approached, and $77 \%$ took part in a health interview. During this interview, respondents reported acute complaints and chronic disorders and they completed a number of questionnaires. Mental distress was operationalized as the report of any of the seven acute mental complaints indicative of mental distress listed in Table 1. These data constitute dataset 1.

\section{Dataset 2}

Data were also collected about doctor-patient contacts over a 3 -month period. During this period. GPs registered all doctor-patient encounters in their practices, thereby providing information about patients' demand for help and the GPs' diagnoses, both coded according to the International Classification of Primary Care. This classification allows the GP to classify symptoms of the patient as well as diagnoses in 18 chapters, according to their anatomical and physiological characteristics. One chapter is devoted to psychological symptoms and diagnoses. Most of the chapters have a code for "fear of ...": for instance. K24 "fear of heart attack" and D26 "fear of stomach cancer." Each diagnosis was accompanied by an assessment of other possible diseases or conditions as explanations for the symptoms. For instance, eye symptoms leading to a diagnosis of retinopathy could be further explained by diabetes as background disease. In total, 377,855 consultations were recorded. Relevant variables are given in Table II. 
Table I.-Dataset 1: Relevant variables measured during health interview $(n=10,787)$

Sociodemographic variables

Age

Gender

Health locus of control questionnaire

Index of mental distress

Patient reports any of the following symptoms during the past 14 days:

Nervousness

Easily agitated

Sleeplessness

Listlessness

Problems at work

Family problems

Aggressive feelings

Chronic somatic disease

Patient indicates on questionnaire to suffer from:

Chronic bronchitis

Asthma

Hypertension

Heart disease

Back symptoms

Rheumatoid arthritis

Cancer

Parkinson's disease/epilepsy/multiple sclerosis

Migraine

Diabetes

Digestive disease

Renal disease

Thyroid disorder

Chronic menstrual symptoms

Consequences accident

To make comparisons between patients with different somatic diagnoses, patients were grouped according to a given diagnosis. For example, the consultations of all patients who had a diagnosis of diabetes were collected for the 3 -month period. Not all of these consultations necessarily concerned diabetes. The same procedure was followed for patients with other diseases mentioned in the questionnaire of dataset 1 . In some cases (renal disease, thyroid disorder, and trauma after accident) there were too few patients with these diagnoses. In the case of back symptoms (the term used in the questionnaire), we decided to distinguish between diagnoses made at the symptom level (low back pain without radiation)

Table II.-Dataset 2: 377,855 consultations by 166,443 patients

For each doctor-patient contact

Sociodemographic variables

Age

Gender

Symptoms, complaints and reasons for visit, as stated by the patient (classified in the International Classification of Primary Care ICPC)

Symptoms classified within chapter P (psychological)

Symptoms classified within different chapters, concerning fear or anxiety about (the consequences of) disease

Diagnoses made by the GP (classified in the International Classification of Primary Care, ICPC), among which there were:

Diagnoses within chapter P (psychological)

Diagnoses with the assessment: complaints due to family problems

Diagnoses with the assessment: complaints due to depression 
and established clinical diagnoses, such as slipped lumbar discs. We also distinguished between rheumatoid arthritis and accompanying diagnoses such as spondylitis and osteoarthritis separately, (these were handled separately in the questionnaire). Thus, we distinguished between the following diseases and symptoms: diabetes, any diagnosis of cancer, heart diseases, neurological diseases (Parkinson's disease. multiple sclerosis, and epilepsy), migraine, respiratory diseases (asthma, chronic bronchitis and emphysema), digestive disease (gastroenteritis, gastric ulcers, etc.), rheumatoid arthritis, chronic diseases of the back (slipped lumbar disc, etc.), symptoms of the back, and symptoms of menstrual problems. The last two categories can be considered as chronic conditions at the symptom level instead of diseases with a known etiology.

Dependent variables for each group of patients with a specific somatic diagnosis were the proportion of patients with at least one diagnosis of psychological disorder, the proportion of patients with at least one psychological complaint as reason for visiting the GP, the proportion of patients whose complaints were attributed to family problems by their GP, the proportion of patients whose complaints were attributed to depression, and the proportion of patients who had mentioned fears and anxieties about their diseases.

The number of patients included in each disease category varied from approximately 1240 patients with migraine to more than 7000 patients with heart disease.

\section{Analysis}

Odds ratios for reporting mental distress were calculated for patients with each chronic condition, using dataset 1 . The odds ratios were calculated in a logistic regression model and were controlled for age and gender. This procedure is considered necessary as psychological distress as well as most somatic diseases are associated with age and gender [24]. At first, all patients with a disease were compared with all patients not reporting that disease. As chronic diseases seldom occur in isolation, many patients reported suffering from more than one chronic condition. To estimate the effect of one single disease as accurately as possible, the analyses were repeated for patients reporting only one chronic disease.

By and large, the same procedure was followed with respect to the doctor-patient contacts during the 3-month period (dataset 2). In this case, patients with 1 of the 11 somatic diagnoses were compared with the complementary group of patients who were not included in that specific disease category. Odds ratios were calculated for patients presenting with psychological complaints; the symptoms and reason for visit. for patients presenting with fears and anxiety about somatic disease; for diagnosis within the psychological chapter of the ICPC; for the GP's suspicion that the complaints were related to depression or family problems. Again, age and gender were included as control variables. As data for each disease category were compared with their complement, the reference group was different for each of the 11 analyses. To aid interpretation of the results, so that all disease categories were compared with the same reference group, the patients of each disease category were also compared with the total population of $166.443 \mathrm{pa}$ tients.

\section{RESULTS}

The data collected during the health interview are given in Table III. Thirty-eight percent of the respondents reported experiencing at least one psychological symptom during the past 14 days, and $55 \%$ of the respondents reported suffering from at least one chronic condition. The most frequently reported conditions were chronic back symptoms, migraine, hypertension, chronic bronchitis, and heart disease. As can be seen in Table III, very few patients reported only one chronic condition. The odds ratios for the recent experience of psychological symptoms increased markedly with the number of chronic conditions (Fig. 1). The odds ratios for people who did not report having a chronic disease were about five times lower than the odds ratios for people who reported three or more chronic diseases.

The odds ratios for recent experience of psychological symptoms for all patients reporting a chronic condition and for patients who reported only one chronic condition are given in Table IV. Again, the presence of a chronic disorder was associated with mental distress. For example, patients suffering from heart disease were 2.65 times more likely to report acute psychological complaints, such as depression or anxiety, than patients without heart disease. For some disorders the odds ratios 
Table III.-Distribution of the variables in dataset 1

\begin{tabular}{|c|c|c|}
\hline & Number of patients $(\%)$ & Number of patients \\
\hline \multicolumn{3}{|l|}{ Age range } \\
\hline $15-24$ & $2108(20 \%)$ & \\
\hline $25-44$ & $4538(42 \%)$ & \\
\hline $45-64$ & $2733(25 \%)$ & \\
\hline $65-74$ & $943(9 \%)$ & \\
\hline $75-98$ & $465(4 \%)$ & \\
\hline \multicolumn{3}{|l|}{ Gender } \\
\hline Male & $5284(49 \%)$ & \\
\hline Female & $5503(51 \%)$ & \\
\hline \multicolumn{3}{|l|}{ Psychological symptoms last 14 days } \\
\hline No & $6736(62 \%)$ & \\
\hline Yes & $4051(38 \%)$ & \\
\hline \multicolumn{3}{|l|}{ Chronic conditions } \\
\hline Chronic bronchitis & $595(6 \%)$ & 142 \\
\hline Asthma & $223(2 \%)$ & 37 \\
\hline Hypertension & $966(9 \%)$ & 292 \\
\hline Heart disease & $595(6 \%)$ & 93 \\
\hline Back symptoms/diseases & $1571(15 \%)$ & 457 \\
\hline Rheumatoid arthritis & $373(3 \%)$ & 62 \\
\hline Cancer & $110(1 \%)$ & 21 \\
\hline Migraine/chronic headache & $1119(10 \%)$ & 335 \\
\hline Parkinson/epilepsy/multiple sclerosis & $143(1 \%)$ & 36 \\
\hline Diabetes & $234(2 \%)$ & 50 \\
\hline Digestive disease & $452(4 \%)$ & 88 \\
\hline Renal disease & $177(2 \%)$ & 34 \\
\hline Thryoid disorder & $134(1 \%)$ & 25 \\
\hline Chronic menstrual symptoms & $357(3 \%)$ & 101 \\
\hline Consequences accident & $290(3 \%)$ & 93 \\
\hline
\end{tabular}

${ }^{a}$ Number of persons with the chronic condition as the only chronic condition.

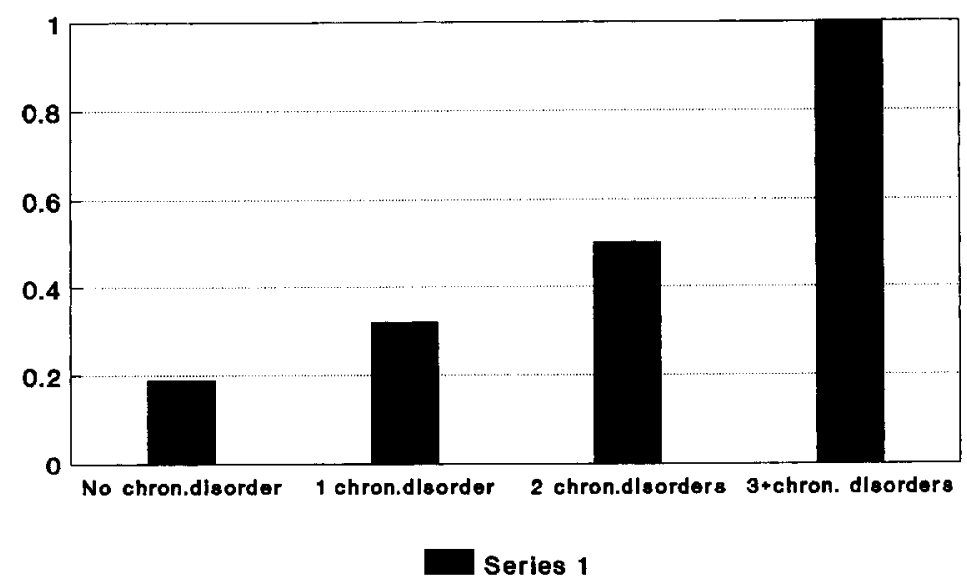

(adjusted for age and $s e x$ )

Fig. 1. Odds on acute psychological symptoms (reference: 3 or more disorders). 
Table IV._Odds ratios (OR) for acute psychological symptoms for patients with and without specific chronic diseases (adjusted for age and gender)

\begin{tabular}{|c|c|c|c|c|}
\hline & \multicolumn{2}{|c|}{ All patients } & \multicolumn{2}{|c|}{$\begin{array}{l}\text { Patients with one } \\
\text { specific chronic disease }\end{array}$} \\
\hline & OR & $\begin{array}{l}0.95 \text { Confidence } \\
\text { interval }\end{array}$ & $\mathrm{OR}$ & $\begin{array}{l}0.95 \text { Confidence } \\
\text { interval }\end{array}$ \\
\hline Chronic bronchitis & 1.57 & $(1.32-1.86)$ & 1.09 & $(0.77-1.57)$ \\
\hline Asthma & 1.94 & $(1.48-2.55)$ & 1.34 & $(0.68-2.66)$ \\
\hline Hypertension & 1.76 & $(1.53-2.03)$ & 1.51 & $(1.18-1.94)$ \\
\hline Heart disease & 2.65 & $(2.21-3.16)$ & 1.87 & $(1.23-2.86)$ \\
\hline Back symptoms & 2.23 & $(2.00-2.50)$ & 1.59 & $(1.30-1.94)$ \\
\hline Rheumatoid arthritis & 2.23 & $(1.79-2.78)$ & 1.83 & $(1.10-3.04)$ \\
\hline Cancer & 1.80 & $(1.22-2.65)$ & 1.65 & $(0.69-3.96)$ \\
\hline Neurological disease ${ }^{a}$ & 2.47 & $(1.75-3.47)$ & 1.69 & $(0.87-3.31)$ \\
\hline Migraine & 2.61 & $(2.30-2.97)$ & 1.67 & $(1.34-2.09)$ \\
\hline Diabetes & 1.66 & $(1.27-2.17)$ & 1.25 & $(0.70-2.25)$ \\
\hline Digestive disease & 2.73 & $(2.28-3.38)$ & 1.52 & $(0.98-2.36)$ \\
\hline Renal disease & 2.06 & $(1.52-2.79)$ & 1.47 & $(0.73-2.96)$ \\
\hline Thryoid disorder & 2.48 & $(1.72-3.57)$ & 1.64 & $(0.74-3.65)$ \\
\hline Chronic menstrual symptoms & 2.38 & $(1.91-2.97)$ & 1.84 & $(1.24-2.73)$ \\
\hline Consequences accident & 1.75 & $(1.38-2.22)$ & 1.20 & $(0.77-1.87)$ \\
\hline
\end{tabular}

"Parkinson's disease, epilepsy, multiple sclerosis.

were higher than for other disorders, but they were all $>1$ and were statistically significant.

When we calculated odds ratios for those people who reported only one chronic disease, a different picture arose. All odds ratios declined and the number of significant odds ratios was lower. Chronic conditions on a symptom level, such as hypertension, back symptoms, and chronic menstrual complaints, were associated with a significantly greater chance of reports of acute psychological complaints. These conditions are less life threatening and definite than real diseases. The odds ratios associated with cancer, asthma, diabetes, neurological disease. or arthritis were no longer statistically significant. Perhaps the former significant results can be attributed to comorbidity. However, all odds ratios were $>1$; in addition, because the conditions that were significantly associated with mental distress were also the most frequently mentioned conditions, it might as well be possible that we have an artifact of statistical power. When these analyses were repeated without controlling for age and gender, some of the odds ratios increased but not statistically significantly $(p>0.05)$.

For the next analyses, data on doctor-patient contacts were used (dataset 2) (Table $\mathrm{V}$ ). About $12 \%$ of the patients complained about psychological symptoms during the 3-month period. A relatively small group expressed fears or anxiety about the consequences of their disease $(2 \%)$. Thirteen percent of the patients were given a psychological diagnosis, such as depression, anxiety disorder, and stress, by their GP. In $2 \%$ the GP attributed the symptoms of the patient to depression and in $5 \%$ to family problems.

The odds ratios for psychological demands for help and fear of illness of patients with and without specific diseases are given in Table VI. Most patients with one of the listed diseases or disorders mentioned psychological reasons more than patients 
Table V.-Distribution of variables in dataset 2: 166,443 patients

\begin{tabular}{lc}
\hline & Number of subjects (\%) \\
\hline Dependent variables & \\
Psychological symptoms or complaints (ICPC, chapter P) & $19,444(12 \%)$ \\
Symptoms/complaints of fear of (consequences of) disease & $2923(2 \%)$ \\
Diagnosis with ICPC chapter P & $21,537(13 \%)$ \\
Symptoms attributed to depression & $2815(2 \%)$ \\
Symptoms attributed to family problems & $7489(5 \%)$ \\
Independent variables & \\
Age range & \\
$0-14$ & $26,199(16 \%)$ \\
$15-24$ & $25,736(16 \%)$ \\
$25-44$ & $50,979(31 \%)$ \\
$45-64$ & $35,372(21 \%)$ \\
$65-74$ & $15,187(9 \%)$ \\
$75-98$ & $12,898(8 \%)$ \\
Gender & \\
Male & $69,502(42 \%)$ \\
Female & $96,941(58 \%)$ \\
Somatic diagnoses & \\
Diabetes & 3591 \\
Any diagnosis of cancer & 1690 \\
Heart diseases & 7196 \\
Neurological diseases & 1362 \\
Migraine & 1240 \\
Rheumatoid arthritis & 3165 \\
Asthma, chronic bronchitis, emphysema & 5344 \\
Any digestive disease & 5087 \\
Chronic diseases of the back & 3525 \\
Symptoms of the back & 1351 \\
Symptoms of menstrual problems & 1974 \\
\hline
\end{tabular}

Table VI.-Odds ratios (OR) for psychological demands for help and on demands for help for fear of illness in patients with and without specific chronic diseases (adjusted for age and gender)

\begin{tabular}{lccccc}
\hline & \multicolumn{2}{c}{$\begin{array}{c}\text { Psychological demands for } \\
\text { help }\end{array}$} & & \multicolumn{2}{c}{$\begin{array}{c}\text { Fear of (consequences of) } \\
\text { disease }\end{array}$} \\
\cline { 2 - 3 } \cline { 5 - 6 } & OR & $\begin{array}{c}0.95 \text { Confidence } \\
\text { interval }\end{array}$ & & OR & $\begin{array}{c}\text { 0.95 Confidence } \\
\text { interval }\end{array}$ \\
\hline Diabetes & 0.82 & $(0.75-0.89)$ & & 0.80 & $(0.62-1.04)$ \\
Any diagnosis of cancer & 1.10 & $(0.97-1.24)$ & & 1.96 & $(1.52-2.53)$ \\
Heart diseases & 1.06 & $(0.99-1.12)$ & & 1.01 & $(0.85-1.20)$ \\
Neurological diseases & 1.49 & $(1.30-1.71)$ & & 0.50 & $(0.29-0.87)$ \\
Migraine & 1.39 & $(1.19-1.62)$ & & 0.78 & $(0.49-1.25)$ \\
Rheumatoid arthritis & 0.96 & $(0.88-1.05)$ & & 1.11 & $(0.87-1.40)$ \\
Asthma, chronic bronchitis, & & & & & \\
$\quad$ emphysema & 1.00 & $(0.93-1.09)$ & & 0.82 & $(0.65-1.02)$ \\
Any digestive disease & 1.27 & $(1.17-1.37)$ & & 1.90 & $(1.63-2.23)$ \\
Chronic diseases of the back & 0.84 & $(0.76-0.93)$ & & 1.38 & $(1.11-1.71)$ \\
Symptoms of the back & 1.19 & $(1.02-1.39)$ & & 1.49 & $(1.06-2.07)$ \\
Symptoms of menstrual problems & 1.30 & $(1.14-1.47)$ & & 1.36 & $(1.03-1.81)$ \\
\hline
\end{tabular}


Table VII.-Odds ratios (OR) for psychological diagnoses by GP

\begin{tabular}{lcc}
\hline & OR & $\begin{array}{c}0.95 \text { Confidence } \\
\text { interval }\end{array}$ \\
\hline Diabetes & 0.74 & $(0.67-(0.81)$ \\
Any diagnosis of cancer & 0.84 & $(0.74-0.95)$ \\
Heart diseases & 0.97 & $(0.91-1.03)$ \\
Neurological discases & 1.30 & $(1.13-1.49)$ \\
Migraine & 1.14 & $(0.98-1.33)$ \\
Rheumatoid arthritis & 0.89 & $(0.82-0.98)$ \\
Asthma, chronic bronchitis, emphysema & 0.94 & $(0.87-1.02)$ \\
Digestive diseases & 1.14 & $(1.05-1.23)$ \\
Chronic diseases of the back & $(0.78$ & $(0.70-(0.86)$ \\
Symptoms of the back & 1.08 & $(0.93-1.25)$ \\
Symptoms of menstrual problems & 0.99 & $(0.87-1.13)$ \\
\hline
\end{tabular}

without the disease. Patients with back symptoms, menstrual symptoms, migraine, digestive diseases, and neurological diseases made significantly more psychological complaints than did the average patient from the total population. Patients with diabetes and chronic diseases of the back, such as slipped lumbar discs, were less likely to have psychological symptoms than the average patient. Patients with cancer. heart diseases, asthma, and rheumatoid diseases did not differ significantly from other patients in their psychological symptoms.

Patients with cancer and diseases of the back, however, were more likely to present with fears or anxiety about disease or the consequences of disease than other patients. Patients with digestive diseases and patients with symptom diagnoses of the back and of menstrual problems also presented with these fears relatively frequently. Patients with neurological diseases mentioned these fears relatively rarely. Patients with the other diseases did not mention fears and anxiety more often than did the average patient.

Table VII presents the odds ratio of a person being given a psychological diagnosis. This was higher for patients with a neurological disease or a digestive disease. In contrast, the odds ratios were significantly lower for patients with diabetes, cancer, rheumatoid diseases, and chronic diseases of the back. Statistically significant odds ratios were not found for the other conditions.

Evaluation of the chance that a GP considered depression to explain a patient's symptoms (Table VIII) gave results similar to those listed in Table VI for the psychological symptoms of the patients. A GP seldom considered family problems to be the cause of the complaints of patients with severe chronic diseases such as diabetes. cancer. and heart disease, whereas the opposite was true for patients with symptom diagnoses (back symptoms, migraine, menstrual complaints), digestive diseases, and chronic diseases of the back.

As mentioned in the Methods section, all analyses on dataset 2 were repeated by comparing data for patients with a given disease with all patients. The outcomes were comparable with the results given in Tables IV-VI. In summary, diabetes, heart disease, rheumatoid diseases, cancer, and lung diseases were not associated with mental distress, except in some cases, with fear or anxiety about the disease. Neurological and digestive diseases were, in all respects. associated with mental distress. Back symptoms, menstrual symptoms, and migraine were associated with psy- 
Table VIII.-Odds ratios (OR) for psychological attributions of the GP to the symptoms of the patient (adjusted for age and gender)

\begin{tabular}{|c|c|c|c|c|}
\hline & \multicolumn{4}{|c|}{ Symptoms attributed to: } \\
\hline & \multicolumn{2}{|r|}{ Depression } & \multicolumn{2}{|c|}{ Family problem } \\
\hline & $\mathrm{OR}$ & $\begin{array}{l}0.95 \text { Confidence } \\
\text { interval }\end{array}$ & OR & $\begin{array}{l}0.95 \text { Confidence } \\
\text { interval }\end{array}$ \\
\hline Diabetes & 0.53 & $(0.41-0.69)$ & 0.52 & $(0.43-0.64)$ \\
\hline Cancer & 0.85 & $(0.62-1.16)$ & 0.61 & $(0.43-0.77)$ \\
\hline Heart diseases & 0.68 & $(0.57-0.80)$ & 0.48 & $(0.41-0.56)$ \\
\hline Neurological diseases & 1.48 & $(1.09-2.02)$ & 0.81 & $(0.61-1.07)$ \\
\hline Migraine & 2.03 & $(1.51-2.74)$ & 2.08 & $(1.72-2.51)$ \\
\hline Rheumatoid arthritis & 1.00 & $(0.82-1.22)$ & 0.80 & $(0.67-0.95)$ \\
\hline Asthma, chronic bronchitis, emphysema & 0.79 & $(0.63-0.99)$ & 0.71 & $(0.61-0.83)$ \\
\hline Any digestive diseases & 2.42 & $(2.10-2.79)$ & 2.68 & $(2.45-2.94)$ \\
\hline Chronic diseases of the back & 1.23 & $(0.99-1.52)$ & 1.18 & $(1.02-1.37)$ \\
\hline Symptoms of the back & 1.86 & $(1.39-2.52)$ & 1.72 & $(1.40-2.10)$ \\
\hline Symptoms of menstrual problems & 1.59 & $(1.22-2.07)$ & 1.96 & $(1.69-2.27)$ \\
\hline
\end{tabular}

chological symptomatology and the GP's suspicion that the patient's complaints had a psychological background but not with psychological diagnoses.

\section{DISCUSSION}

By and large, we found a clear relationship between somatic disease and mental distress, as indicated by patient self-report data, by the presentation of psychological complaints when patients visited their GPs, and by the diagnosis of the GP. However, this relationship was weak, absent, or even the other way around for the most severe or disabling diseases, which can be objectified, such as cancer, arthritis, or diabetes. It is striking that this was also true for diseases such as diabetes, which is prototypical of a disease that can be controlled, as well as for cancer and arthritis, which are prototypical for less controllable diseases, because it has been hypothesized that diseases which are beyond the control of the patient especially act as stressors and cause psychological problems [25].

The relationship between disease and mental distress was strong for the neurological diseases Parkinson's disease, multiple sclerosis, and epilepsy, diseases for which direct biological links between the disease and mental distress have been demonstrated. The relationship was also strong for digestive diseases and for a number of diagnoses that are not actual diseases but symptom diagnoses, such as back symptoms and menstrual symptoms, and for migraine. For these symptom diagnoses, it was not the diagnosis of a psychological disorder by the GP, but the psychological complaints and the GP's suspicion of there being a psychological background to the complaints which were related to the physical symptoms.

Our results are consistent with earlier findings, mentioned in the Introduction section, although the results concerning "classical" chronic somatic diseases need to be discussed further. Relatively high prevalences of mental distress have been repeatedly reported in diabetes, cancer, lung diseases, heart diseases, neurological diseases, and arthritis [26]. It should be emphasized, however, that in these cases distress was assessed on the basis of questionnaires or diagnostic interviews. Patients' 
demand for help was not taken into consideration. Measuring patients' experience of distress, by questionnaire, we found comparable results, although also on that occasion the less severe "symptomatic" chronic conditions had the highest odds ratios and the more severe diseases did not reach the level of significance, when inspected scrupulously. When, however, the demand for help, as put forward during the doctor-patient contact, and the resulting diagnoses were taken into consideration. patients with "classical" chronic diseases did not complain more and were not given a psychological diagnosis more frequently than other patients.

With regard to the possible explanations for the relationship between somatic disease and psychological complaints, our results provide evidence for the hypothesis that psychological distress is manifested by physical symptoms. Symptom diagnoses, gastrointestinal diseases, and migraine were typically associated with psychological distress. The explanation that disease itself is a stressor, and especially life-threatening or disabling diseases, such as arthritis, cancer, or heart disease, could not be proven when patients' demands for help or GPs' diagnoses were taken into consideration. The explanation that there is a direct biological link between somatic and psychological disorders appeared to be valid for neurological diseases indeed, as was to be expected from the literature. The suggestion that comorbidity of somatic disease and mental disorder, perhaps mediated by increasing age, can explain the relationship between somatic disease and psychological distress could not be confirmed, because the same results were obtained when analyses were controlled. or not, for age and gender.

It is important to consider the nature of our data. We used patients' self-report data and registration and evaluation by their GPs. A plausible interpretation is that symptoms of distress of patients who are seriously ill are more easily interpreted (by GP and patient) as understandable consequences of their illness and thus not labeled as psychological symptoms. However, this should not be viewed as measurement error. It is significant for the meaning GP and patient attach to the symptoms. given the context of severe somatic disease. When psychiatric epidemiologists conduct interviews, they make note of these symptoms and arrive at a psychiatric diagnosis, apart from this context, whereas a treating doctor considers the symptoms as part of the somatic disease.

An important question in this respect is how the patient evaluates these symptoms. On the basis of their demands for help (as registered by the GPs!), patients with serious diseases did not ask for help for their psychological symptoms, although they sometimes needed help for their fear of the consequences of disease. It is possible that GPs do not recognize this problem. Whether underrecognition occurs can be assessed, not by questioning the patient out of the context of consultation, but by examining the consultations in more detail. Research by means of videotaped observations has proven helpful in the past $[27,28]$. It is important that possible underestimation by GPs becomes clear because there are a number of interventions for providing psychological help to patients whose demand for help is not recognized by the GP [26]. This type of research could inform us about the magnitude of underestimation of patients' demands and provide guidelines to be incorporated into graduate and postgraduate training programs.

If, on the other hand, the patient generally experiences his or her adaptive tasks. not so much as an extra psychological problem but as one associated with his or her 
disease, then a good integration of somatic and psychosocial care in the treatment of the disease is needed.

Stronger relationships could be found between psychological distress (in all operationalizations) and less serious illnesses, leading us to the conclusion that a substantial part of the relationship between somatic disease and psychological disorder can be traced back to this association. These patients have long been an object of concern of medical doctors, and a whole research tradition on somatization is devoted to them [13]. A combined approach of attention to the physical complaints, without unnecessary diagnostics and treatment, and investigation of the possible psychological backgrounds is recommended.

\section{REFERENCES}

1. Bensing JM, Verhaak PFM. Psychische problemen in de huisartspraktijk veelvormiger en diffuser dan in de psychiatrie [Mental problems in general practice more variable and diffuse than in psychiatry]. Nederlands Tijdschrift voor Geneeskunde 1994;138:130.

2. Schulberg HC, McClelland M, Burns BJ. Depression and physical illness: the prevalence, causation, and diagnosis of comorbidity. Clin Psychol Rev 1987;7:145.

3. Wells KB, Stewart AL, Hays RD, Burnam MA, Rogers WH, Daniels M, Berry SD, Greenfield S, Ware JE. The functioning and well-being of depressed patients. Results from the medical outcomes study. JAMA 1989;262:914.

4. Wilkinson G, Smeeton H, Skuse D, Fry J. Consultation for physical illnesses by patients diagnosed and treated for psychiatric disorders by a general practitioner: 20 years following study. BMJ 1988;297:776.

5. Hall RCW, Popkin MK, Devaul RA, Faillace LA, Stickney SK. Physical illness presenting as psychiatric disease. Arch General Psychiat 1978;35:1315.

6. Hall RCW, Gardner ER, Stickney SK, LeCann AF, Popkin MK. Physical illness manifesting as psychiatric disease. II. Analysis of a state hospital inpatient population. Arch General Psychiat 980:37:989.

7. Fichter MM, Rehm J, Elton M, Dilling H, Achatz F. Mortality risk and mental disorder: Iongitudinal results from the Upper Bavarian Study. Psychol Med 1995;25:297.

8. Huppert FA, Whittington JE. Symptoms of psychological distress predict 7-year mortality. Psychol Med 1995;25:1073.

9. Holland JC, Korzun AH, Tross S, Silberfarb P, Perry M, Comis R, Oster M. Comparative psychological disturbance in patients with pancreatic and gastric cancer. Am J Psychiat 1986;143:982.

10. Popkin MK, Callies AL, Lentz RD, Colon EA, Sutherland DE. Prevalence of major depression, simple phobia, and other psychiatric disorders in patients with long-standing type I diabetes mellitus. Arch Gen Psychiat 1988;45:64.

11. Wells KB, Golding JM, Burnam MA. Psychiatric disorder in a sample of the general population with and without chronic medical conditions. Am J Psychiat 1988;145:976.

12. Stewart AL, Greenfield S, Hays RD, Wells KB, Rogers WH, Berry SD, McGlynn EA. Ware JE. Functional status and well-being of patients with chronic conditions. Results from the medical outcome study. JAMA 1989:262:907.

13. Katon W, Sullivan M. Depression and chronic medical illness. J Clin Psychiat 1990;51(suppl):3.

14. McDaniel JS, Musselman DL, Porter MR, Reed DA, Nemeroff CB. Depression in patients with cancer. Arch Gen Psychiat 1995;52:89.

15. Cassileth BR, Lusk EJ, Strouse TB, Miller DS, Brown LL, Cross PA, Tenaglia AN. Psychosocial status in chronic illness. A comparative analysis of six diagnostic groups. N Engl J Med 1984:311:506.

16. Spitzer RL, Kroenke K, Linzer M, Hahn R, Williams JBW, deGruy FV de, Brody D, Davies M. Health related quality of life in primary care patients with mental disorders. JAMA 1995;274:1511.

17. Brown JH, Paraskevas F. Cancer and depression: Cancer presenting with depressive illness: An auto-immune disease? Br J Psychiat 1982;141:227.

18. Miranda J, Arean PA, Rickman RL. Relationship of mental and medical disorder. In: Miranda J, Hohmann AA, Attkinson CC, Larson DB, eds. Mental disorders in primary care. San Francisco, California: Jossey-Bass 1994.

19. Lipowski ZJ. Somatization and depression. Psychosomatics 1990:31:13.

20. Friedman HS, Booth-Kewley S. The "disease prone personality." Am Psychologist 1987:42:539.

21. Hayward C. Psychiatric illness and cardiovascular disease risk. Epidemiol Rev 95;17:129. 
22. Verbrugge LM. New thinking and science on disability in mid-and late life. Eur $\mathbf{J}$ Pub Health $1995 ; 5: 20$.

23. Mirowski J, Ross CE. Age and depression. J Health Social Behav 1992;33:187.

24. Verhaak PFM. Mental disorder in the community and in general practice. Avehurv. UK: Aldershot 1995.

25. Feifel H, Strack S, Tong Nagy V. Degree of life threat and differential use of coping modes. J Psychosom Res 1987;31:91.

26. Poppelaars CAM, Kaptein AA. Psychologische en psychiatrische problematiek bij chronisch somatisch zieken. Overzichtsstudie [Psychological and psychiatric problems in chronic somatic diseases. An overview]. Zoetermeer: Nationale Commissie Chronisch Zieken 1994.

27. Bensing JM. Doctor-patient communication and the quality of care. PhD thesis. Utrecht: NIVEL 1991.

28. Verhaak PFM. Detection of psychological disorder by general practitioners. Med Care 1988:26:10004. 